\title{
Simulation of electron trajectories in nuclear emulsion and its application
}

\author{
Atsushi lyono ${ }^{* a}$, Saya Yamamoto ${ }^{b}$, Hiroki Matsumoto ${ }^{c}$, Kazuhide Okei ${ }^{c}$ and Takao \\ Nakatsuka ${ }^{d}$, \\ ${ }^{a}$ Dept. of Fundamental Science, Faculty of Science, Okayama university of Science \\ 1-1 Ridai-cho, Kita-ku, Okayama-city, Okayama 700-0005, Japan \\ ${ }^{b}$ Graduate School of Science, Okayama University of Science \\ 1-1 Ridai-cho, Kita-ku, Okayama-city, Okayama 700-0005, Japan \\ ${ }^{c}$ Kawasaki Medical School \\ 577 Matsushima, Kurashiki-city, Okayama 701-0192, Japan \\ ${ }^{d}$ Okayama Shoka University \\ 2-10-1Tsushima Kyomachi, Kita-ku, Okayama-city ,Okayama 700-8601, Japan \\ E-mail: \\ iyono@das.ous.ac.jpls15sm08@ous.jplh.mat sumoto@med.kawasaki-m.ac.jp \\ okei@med.kawasaki-m.ac.jplnakatuka@olive.plala.or.jp
}

\begin{abstract}
Energies of charged particle are determined in nuclear emulsion experiments by tracing trajectories of charged particle and analyzing their multiple scattering properties. Traditionally they use multiple scattering theory under the gaussian approximation and determine the energies from the mean square deflection angle. Multiple scattering theory is improved today to take account the single and the plural scatterings together, where the mean square deflection angle almost diverges. We examine feasibility of the most likelihood method with Moliere theory of multiple scattering to determine the energy of charged particle. We also examine Moliere simultaneous distribution to determine the energy more accurately.
\end{abstract}

The 34th International Cosmic Ray Conference,

30 July- 6 August, 2015

The Hague, The Netherlands

\footnotetext{
*Speaker.
} 


\section{Introduction}

The energy or the momentum of charged particles has been determined by angular or sagitta method in nuclear emulsion experiments [1], tracing trajectories of charged particle and comparing those with multiple scattering theory. DONUT project applied the angular method in his nutrino experiment, where he applied the multiple scattering theory under the gaussian approximation and determined the momentum of charged particles from the mean square deflection angle [2].

The multiple scattering theory is improved today to take account the single and the plural scatterings other than the multiple scattering, where the mean square deflection angle diverges so that the momentum of charged particle cannot be determined by the traditional method any more. We examine another angular method to determine the energy of charged particles by the improved multiple scattering theory of Moliere, and further attempt to apply the Moliere simultaneous distribution to determine the energy of charged particles more accurately.

\section{Moliere simultaneous distribution between the deflection angle and the lateral displacement}

\subsection{Fourier spectral density}

Charged particles traversing through matters of thickness $t$ in radiation length [3] receive inumerable number of Coulomb scattering, so that they change their deflection angle $\theta$ and lateral displacement $y$ of projected components. Let $f(\theta, \psi, t)$ be the simultaneous probability density between the deflection angle $\theta$ and the chord-angle $\psi$ defined as

$$
\psi \equiv y / t
$$

and $\tilde{f}(\zeta, \eta t, t)$ be its Fourier spectral density

$$
f(\theta, \psi, t) d \theta d \psi=\frac{d \theta d(y / t)}{2 \pi} \iint e^{-i \theta \zeta-i \psi(\eta t)} \tilde{f}(\zeta, \eta t, t) d \zeta d(\eta t),
$$

then $\tilde{f}(\theta, \psi, t)$ is solved as 44

$$
\tilde{f}(\zeta, \eta t, t)=\frac{1}{2 \pi} \exp \left[\int_{0}^{1} \frac{K^{2} t(\zeta+\eta t u)^{2}}{4 \Omega(E+\varepsilon t u)^{2}} \ln \frac{K^{2}(\zeta+\eta t u)^{2}}{4 e^{\Omega}(E+\varepsilon t u)^{2}} d u\right],
$$

where $K$ and $\Omega$ denote the scattering constants introduced by Kamata and Nishimura [6, 7, 4] and $\varepsilon$ the critical energy [3].

Especially under the fixed energy condition $(\varepsilon=0)$, we have

$$
\begin{aligned}
\ln 2 \pi \tilde{f} & =\frac{1}{\Omega} \frac{K^{2} t / E^{2}}{12 \eta t}\left\{(\zeta+\eta t)^{3} \ln \frac{\left(K^{2} / E^{2}\right)(\zeta+\eta t)^{2}}{4 e^{2 / 3+\Omega}}-\zeta^{3} \ln \frac{\left(K^{2} / E^{2}\right) \zeta^{2}}{4 e^{2 / 3+\Omega}}\right\} \\
& =\frac{1}{B} \frac{\theta_{\mathrm{M}}^{2}}{12 \eta t}\left\{(\zeta+\eta t)^{3} \ln \frac{\theta_{\mathrm{M}}^{2}(\zeta+\eta t)^{2}}{4 e^{2 / 3+B}}-\zeta^{3} \ln \frac{\theta_{\mathrm{M}}^{2} \zeta^{2}}{4 e^{2 / 3+B}}\right\},
\end{aligned}
$$

with

$$
\begin{aligned}
& B-\ln B=\Omega-\ln \Omega+\ln t, \\
& \theta_{\mathrm{M}}^{2}=(B / \Omega) K^{2} t / E^{2} .
\end{aligned}
$$

Note that the logarithm of our spectral densiry (2.4) is identical with Molière's result in 1955 [8], where we should remind his $\chi_{c}^{\prime} \sqrt{l_{0} B}$ agrees with our $\theta_{\mathrm{M}}$. 


\subsection{Simultaneous probability density}

As it holds

$$
\tilde{f}(-\zeta,-\eta t, t)=\tilde{f}(\zeta, \eta t, t)
$$

the double Fourier transforms of Eq. (2.2) is reduced to the double cosine transforms as

$$
f(\theta, \psi, t) d \theta d \psi=\frac{d \theta d(y / t)}{\pi} \int_{0}^{\infty} d(\eta t) \int_{-\infty}^{\infty} \cos (\theta \zeta+\psi \eta t) \tilde{f}(\zeta, \eta t, t) d \zeta .
$$

The result of Molière simultaneous distribution between th deflection angle $\theta$ and the chord-angle $\psi$ under the fixed energy condition is indicated in Figs. 1 for $B=8$.

The simultaneous distribution can also be expressed as $g(\rho, \varphi, t) \rho d \rho d \varphi$ in the polar coordinate, satisfying

$$
\begin{aligned}
& f(\theta, \psi, t)=g(\rho, \varphi, t) \quad \text { with } \\
& \theta=\rho \cos \varphi, \quad \psi=\rho \sin \varphi .
\end{aligned}
$$

On the radial axis with the azimuthal angle of 0 , the probability density is expressed as

$$
g(\rho, 0, t)=f(\rho, 0, t)=\frac{1}{\pi} \int_{0}^{\infty} d \zeta \cos (\rho \zeta) \int_{-\infty}^{\infty} \tilde{f}(\zeta, \eta t, t) d(\eta t) .
$$

If we introduce the new $\theta^{\prime}-\psi^{\prime}$ and $\zeta^{\prime}-\eta^{\prime} t$ coordinates by rotating both the $\theta-\psi$ and $\zeta-\eta t$ coordinates with $\varphi$, the probability density on the $\theta^{\prime}$ axis, or $g(\rho, \varphi)$, is derived same way as Eq. 2.11) on the rotated coordinate,

$$
g(\rho, \varphi, t)=\frac{1}{\pi} \int_{0}^{\infty} d \zeta^{\prime} \cos \left(\rho \zeta^{\prime}\right) \int_{-\infty}^{\infty} \tilde{f}\left(\zeta^{\prime} \cos \varphi-\eta^{\prime} t \sin \varphi, \zeta^{\prime} \sin \varphi+\eta^{\prime} t \cos \varphi, t\right) d\left(\eta^{\prime} t\right) .
$$

The radial variations of Molière simultaneous distribution are indicated in Fig. 2 against the azimuthal angles $\varphi$ of $0, \pi / 6, \pi / 3, \pi / 2,2 \pi / 3$, and $5 \pi / 6$ for $B=8$.

\section{Energy determination of charged particle by using the Moliere theory of multiple scattering}

\subsection{Energy decision by the most likelihood method with Moliere angular distribution}

DONUT collaboration determines the momentum of charged particles from the angular distribution of particles in emulsion cloud chamber expected by the multiple scattering theory of gaussian approximation [92]. And MACRO collaboration determines the energy of charged particles from the angular or the lateral distribution of particles in streamer tube chambers expected by the multiple scattering theory of also gaussian approximation [10, 11]. We examine the method to determine the energy of charged particle by using the more accurate theory of multiple scattering by Moliere [12,13,14].

Let $f(\phi, t)$ be the Moliere angular distribution for the projected angle $\theta$ scaled by the Moliere scale angle $\theta_{\mathrm{M}}$ of Eq. [2.6), $\phi \equiv \theta / \theta_{\mathrm{M}}$, irrespective of the projected lateral displacement $y$ [13] 15], then we have

$$
f(\phi, t)=f^{(0)}(\phi)+B^{-1} f^{(1)}(\phi)+B^{-2} f^{(2)}(\phi), \quad 0 \leq \phi
$$




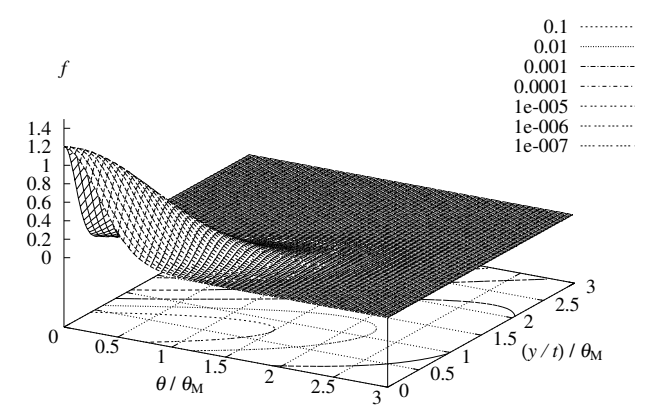

Figure 1: Molière simultaneous distribution expressed by the probability density against the projected deflection angle $\theta$ and the projected chordangle $y / t$, both scaled by $\theta_{\mathrm{M}}$, under the fixed energy condition with $B=8$. Contour map of the density is indicated in the bottom plane.

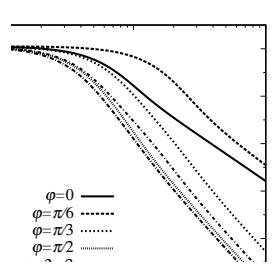

Figure 2: Radial variation of the simultaneous probability density $f(\theta, y / t, t) \equiv g(\rho, \varphi, t)$ for $B=8$ with the radial distance scaled by $\theta_{\mathrm{M}}$, on the azimuthal angles $\varphi$ of $\pi / 6,0, \pi / 3,5 \pi / 6, \pi / 2$, and $2 \pi / 3$ from top to bottom, which shows power decrease with the index about $-4.2,-3.9,-6.2$, $-6.1,-6.0,-6.0$, respectively, from 8 to 10 of the abscissa.

where $f^{(0)}(\phi)=(2 / \sqrt{\pi}) e^{-\phi^{2}}$ and $B$ is determined from $t$ as Eq. (2.5).

Let we get $N$ projected angles

$$
\left\{\theta_{k}\right\} \quad k=1,2, \cdots, N
$$

from $N$ observations. As the probability of appearing projected angle $\theta$ within $d \theta$ is

$$
\theta_{M}^{-1} f\left(\theta / \theta_{M}, t\right) d \theta
$$

mean logarithmic likelihood $L\left(\theta_{M}\right)$ against the scale angle $\theta_{\mathrm{M}}$ is defined as

$$
L\left(\theta_{M}\right)=N^{-1} \sum_{k=1}^{N} \ln \left\{\theta_{M}^{-1} f\left(\theta_{k} / \theta_{M}\right)\right\}
$$

then the scale angle $\theta_{M}$ is determined by the maximal point of $L\left(\theta_{M}\right)$, hence the energy $E$ is determined from Eq. (2.6).

Mean logarithmic likelihood curves $L\left(\theta_{M}\right)$ applied to the Moliere projected-angular distribution $\theta_{M}^{-1} f\left(\theta / \theta_{M}, t\right)$ with $\theta_{M}=1$ are expected as

$$
L\left(\theta_{M}\right)=\int_{0}^{\infty} f(\theta, t) \ln \left\{\theta_{M}^{-1} f\left(\theta_{k} / \theta_{M}\right)\right\} d \theta .
$$

The results for $B=8,12,16$, and $\infty$ are indicate in Fig. 3 All curves have maximul values at about $\theta_{M}=1$, which fact indicates that the most likelihood method with Moliere projected-angular distribution is applicable for energy decision of charged particles traversing through matters.

The maximul feature of the mean logarithmic likelihood $L\left(\theta_{M}\right)$ is more clearly indicated in Fig. 4 by the negative value of relative entropy, $-V$, defined as

$$
-V \equiv \int_{0}^{\infty} f(\theta, t) \ln \frac{\theta_{M}^{-1} f\left(\theta / \theta_{M}, t\right)}{f(\theta, t)} d \theta
$$




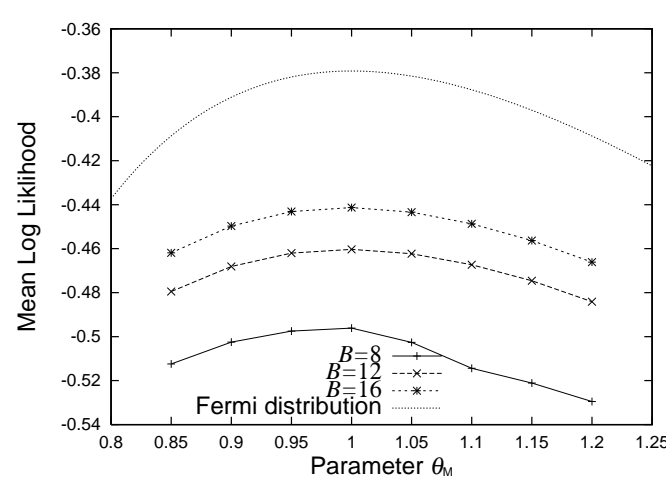

Figure 3: Mean logarithmic likelihood curves $L\left(\theta_{M}\right)$ applied to the Moliere projected-angular distribution of $\theta_{M}^{-1} f\left(\theta / \theta_{M}, t\right)$ with $\theta_{M}=1$, for $B$ of $8,12,16$, and $\infty$ from the bottom to the top.

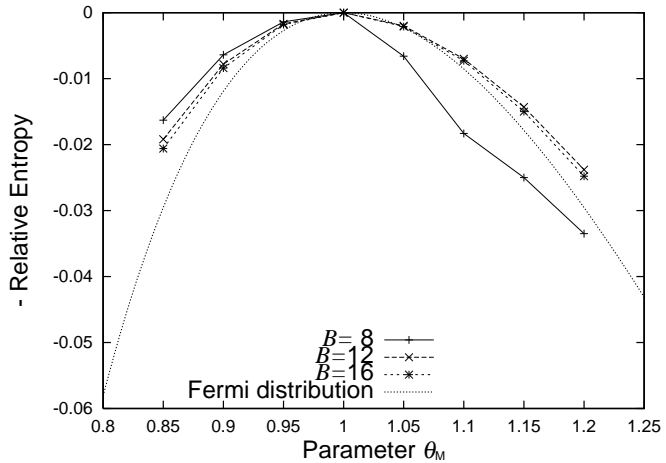

Figure 4: Mean logarithmic likelihood curves expressed by the negative value of the relative entropy, applied to the Moliere projected-angular distribution with $\theta_{M}=1$. Peak values are adjusted to 0 , by substituting constant values indicated in the text.

$$
=\int_{0}^{\infty} f(\theta, t) \ln \left\{\theta_{M}^{-1} f\left(\theta / \theta_{M}, t\right)\right\} d \theta-\int_{0}^{\infty} f(\theta, t) \ln f(\theta, t) d \theta .
$$

\subsection{Energy decision by the most likelihood method with Moliere simultaneous distribution}

We apply Moliere simultaneous distribution between the deflection angle and the lateral displacement $f(\theta, \psi, t)$ or $g(\rho, \varphi, t)$ of Eq. (2.9), instead of the individual distribution for the deflection angle in the preceding subsection, to determine the energy of charged paticles by the most likelihood method.

Let we get $N$ simultaneous data between the deflection projected angle and the lateral displacement (or the chord-angle) of projected components,

$$
\left\{\left(\theta_{k}, \psi_{k}\right)\right\}, \quad k=1,2, \cdots, N
$$

from $N$ observations. As the probability of appearing simultaneous data of projected angle $\theta$ and chord-angle $\psi \equiv y / t$ within $d \theta$ and $d \psi$ is

$$
\theta_{M}^{-2} f\left(\theta / \theta_{M}, \psi / \theta_{M}\right) d \theta d \psi
$$

mean logarithmic likelihood $L\left(\theta_{M}\right)$ against the scale angle $\theta_{\mathrm{M}}$ is defined as

$$
L\left(\theta_{M}\right)=N^{-1} \sum_{k=1}^{N} \ln \left\{\theta_{M}^{-2} f\left(\theta / \theta_{M}, \psi / \theta_{M}\right)\right\}
$$

then the scale angle $\theta_{M}$ is determined by the maximal point of $L\left(\theta_{M}\right)$, hence the energy $E$ is determined from Eq. (2.6). 

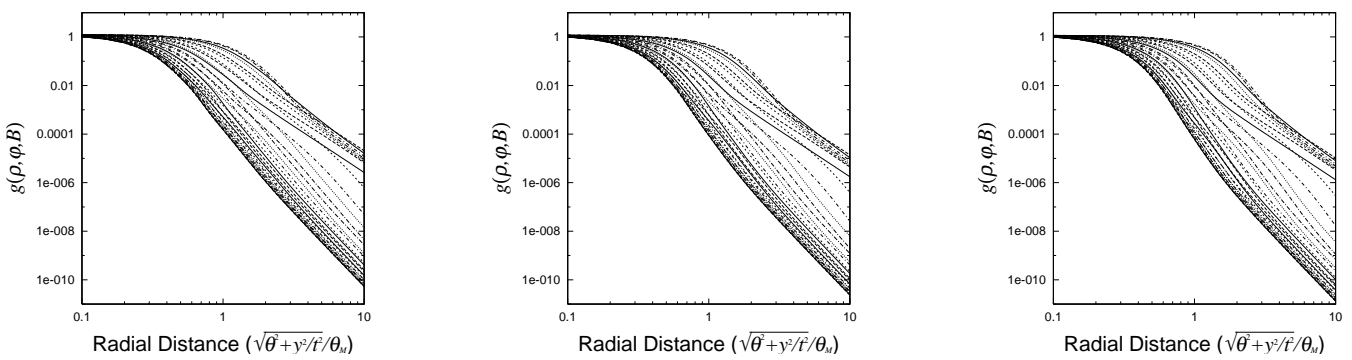

Figure 5: Simultaneous probability desnsity $g(\rho, \varphi)$ for $B=8,12,16$ (left, middle, right). Azimuthal angles are taken as $\varphi_{i}=(i-1) \pi / 30$, with $i=1,2, \cdots, 30$.

Mean logarithmic likelihood curves $L\left(\theta_{M}\right)$ applied to the Moliere simultaneous projected distribution $\theta_{M}^{-2} f\left(\theta / \theta_{M}, \psi / \theta_{M}\right)$ with $\theta_{M}=1$ are expected as

$$
L\left(\theta_{M}\right)=\int_{-\infty}^{\infty} \int_{-\infty}^{\infty} f(\theta, \psi) \ln \left\{\theta_{M}^{-2} f\left(\theta / \theta_{M}, \psi / \theta_{M}\right)\right\} d \theta d \psi
$$

Prior to the expectation of Eq. (3.10), we prepared detailed numerical results of $g(\rho, \varphi)$ for $B=8,12,16$ and $\varphi$ of 30 directions, as indicated in Fig. 5 The results for $B=8,12,16$, and $\infty$ are indicate in Fig. 6 All curves have maximul values at about $\theta_{M}=1$, which fact indicates that the most likelihood method with Moliere simultaneous distribution is applicable for energy decision of charged particles traversing through matters.

The maximul feature of the mean logarithmic likelihood $L\left(\theta_{M}\right)$ is more clearly indicated in Fig. 7 by the negative value of relative entropy, $-V$, defined as

$$
\begin{aligned}
-V & \equiv 2 \int_{0}^{\pi} d \varphi \int_{0}^{\infty} g(\rho, \varphi) \ln \frac{\theta_{M}^{-2} g\left(\rho / \theta_{M}, \varphi\right)}{g(\rho, \varphi)} \rho d \rho \\
& =2 \int_{0}^{\pi} d \varphi \int_{0}^{\infty} g(\rho, \varphi) \ln \left\{\theta_{M}^{-2} g\left(\rho / \theta_{M}, \varphi\right)\right\} \rho d \rho-2 \int_{0}^{\pi} d \varphi \int_{0}^{\infty} g(\rho, \varphi) \ln g(\rho, \varphi) \rho d \rho .
\end{aligned}
$$

The mean logarithmic likelihood curves show narower peaks in Figs. 6 and 7 than in Figs. 3 and 4 , So we find $\theta_{M}$ thus the energy $E$ of charged particles is more accurately determined by applying the Moliere simultaneous distribution, than applying the individual Moliere distribution for deflection angle.

\section{Conclusion}

We have found the energy of charged particle can be determined by applying the most likelihood method with Moliere multiple scattering theory for angular distribution, taking the single and the plural scatterings together into account. We also have found the energy is more accurately determined by applying the Moliere simultaneous distribution than applying the individual Moliere distribution for deflection angle. 


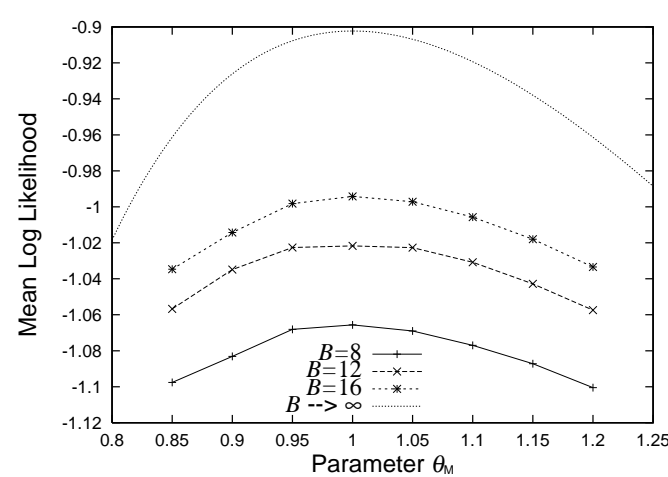

Figure 6: Mean logarithmic likelihood curves $L\left(\theta_{M}\right)$ applied to the Moliere simultaneous projected distribution $\theta_{M}^{-2} f\left(\theta / \theta_{M}, \psi / \theta_{M}\right)$ with $\theta_{M}=1$ for $B=8,12,16$, and $\infty$ from the bottom to the top.

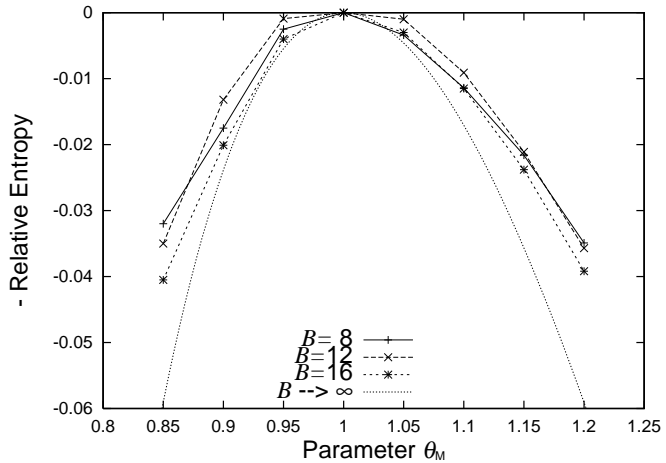

Figure 7: Mean logarithmic likelihood curbes expressed by the negative value of the relative entropy, applied to the Moliere projected-angular distribution with $\theta_{M}=1$. Peak values are adjusted to 0 , by substituting constant values indicated in the text.

\section{References}

[1] P.H. Fowler, Phil. Mag. 41, 169(1950).

[2] M. De Serio, et al., Nucl. Instrum. Methods Phys. Res. A 512, 539(2003).

[3] B. Rossi and K. Greisen, Rev. Mod. Phys. 27, 240(1941).

[4] T. Nakatsuka and J. Nishimura, Phys. Rev. E78, 021136(2008).

[5] K. Okei, T. Nakatsuka, and Y. Kirihara, "Proceedings of the 21st EGS User's Meeting in Japan," KEK Proceedings 2014-6, 1(2014).

[6] K. Kamata and J. Nishimura, Prog. Theor. Phys. Suppl. 6, 93(1958).

[7] J. Nishimura, in Handbuch der Physik, Band 46, edited by S. Flügge (Springer, Berlin, 1967), Teil 2, p. 1.

[8] G. Molière, Z. Naturforsch. 10a, 177(1955).

[9] DONUT Collaboration, K. Kodama, et al., Phys. Lett. B 504, 218(2001).

[10] MACRO Collaboration, M. Ambrosio, et al., Phys. Lett. B 566, 35(2003).

[11] MACRO Collaboration, M. Ambrosio, et al., Nucl. Instrum. Methods Phys. Res. A 492, 376(2002).

[12] G. Molière, Z. Naturforsch. 2a, 133(1947).

[13] G. Molière, Z. Naturforsch. 3a, 78(1948).

[14] H.A. Bethe, Phys. Rev. 89, 1256(1953).

[15] T. Nakatsuka, K. Okei, and N. Takahashi, Nucl. Instrum. Methods Phys. Res. B 311, 60(2013). 